\title{
Theranosties
}

Research Paper

$2011 ; 1: 302-309$

\section{Multimodality Imaging of Tumor Response to Doxil}

\section{Fan Zhang1, 2, Lei Zhu¹, Gang Liư1, Naoki Hida1', Guangming Lư², Henry S. Eden³, Gang Niu1,4凶, Xiaoyuan Chen ${ }^{1 凶}$}

1. Laboratory of Molecular Imaging and Nanomedicine (LOMIN), National Institute of Biomedical Imaging and Bioengineering (NIBIB), National Institutes of Health (NIH), Bethesda, MD 20892, USA

2. Department of Radiology, Nanjing Jinling Hospital, Clinical School of the Medical College of Nanjing University, Nanjing 210002, China

3. Intramural Research Program, National Institute of Biomedical Imaging and Bioengineering, National Institutes of Health, Bethesda, Maryland, 20892, USA

4. Imaging Sciences Training Program, Radiology and Imaging Sciences, Clinical Center and National Institute of Biomedical Imaging and Bioengineering, NIH, 20892, USA

Corresponding author: Dr. Xiaoyuan Chen, 31 Center Drive, Suite 1C14, Bethesda, MD 20892-2281. Email: shawn.chen@nih.gov. Or Dr. Gang Niu, 9 Memorial Drive, 9/1W111, Bethesda, MD 20892. Email: niug@mail.nih.gov

(C) Ivyspring International Publisher. This is an open-access article distributed under the terms of the Creative Commons License (http://creativecommons.org/ licenses/by-nc-nd/3.0/). Reproduction is permitted for personal, noncommercial use, provided that the article is in whole, unmodified, and properly cited.

Received: 2011.05.05; Accepted: 2011.06.01; Published: 2011.06.06

\begin{abstract}
Purpose: Early assessment of tumor responses to chemotherapy could enhance treatment outcomes by ensuring that, from the beginning, treatments meet the individualized needs of patients. In this study, we applied multiple modality molecular imaging techniques to pre-clinical monitoring of early tumor responses to Doxil, focusing on imaging of apoptosis.

Methods: Mice bearing UM-SCC-22B human head and neck squamous cancer tumors received either PBS or I to 2 doses of Doxil ${ }^{\circledR}$ (doxorubicin $\mathrm{HCl}$ liposome injection) (I0 $\mathrm{mg} / \mathrm{kg} /$ dose). Bioluminescence signals from an apoptosis-responsive reporter gene were captured for apoptosis evaluation. Tumor metabolism and proliferation were assessed by ${ }^{18} \mathrm{~F}-\mathrm{FDG}$ and $3^{\prime}$ - $-{ }^{18} \mathrm{~F}$-fluoro-3'-deoxythymidine $\left({ }^{18} \mathrm{~F}-\mathrm{FLT}\right)$ positron emission tomography. Diffusion-weighted magnetic resonance imaging (DW-MRI) was performed to calculate averaged apparent diffusion coefficients (ADCs) for the whole tumor volume. After imaging, tumor samples were collected for histological evaluation, including terminal deoxynucleotidyl transferase dUTP nick end labeling (TUNEL), anti-CD3I, and Ki-67 immunostaining.

Results: Two doses of Doxil significantly inhibited tumor growth. Bioluminescence imaging (BLI) indicated apoptosis of tumor cells after just I dose of Doxil treatment, before apparent tumor shrinkage. ${ }^{18} \mathrm{~F}-\mathrm{FDG}$ and ${ }^{18} \mathrm{~F}-\mathrm{FLT}$ PET imaging identified decreased tumor metabolism and proliferation at later time points than those at which $B L I$ indicated apoptosis. MRI measurements of ADC altered in response to Doxil, but only after tumors were treated with 2 doses. Decreased tumor proliferation and increased apoptotic cells were confirmed by changes of Ki-67 index and apoptotic ratio.

Conclusion: Our study of tumor responses to different doses of Doxil demonstrated that it is essential to combine apoptosis imaging strategies with imaging of other critical biological or pathological pathways, such as metabolism and proliferation, to improve clinical decision making in apoptosis-related diseases and interventions.
\end{abstract}

Key words: Multimodality Imaging, Doxil, Cancer Therapy, Response Monitoring, Apoptosis

\section{Introduction}

It is well known that most chemotherapeutic agents cause tumor cell death primarily by induction of apoptosis, and resistance to anticancer treatment is widely believed to involve mutations that lead to de- 
regulated cellular proliferation and suppression of mechanisms that enable apoptosis [1]. The anthracycline antibiotic doxorubicin, originally isolated from the fungus Streptomyces peucetius, is a chemotherapeutic agent with strong activity against a wide range of human malignant neoplasms [2]. So far, the exact action mechanism of doxorubicin is unknown, but is believed to be related to several pathways. First, doxorubicin intercalates between adjacent DNA base pairs, which may prevent DNA replication and transcription. Second, the drug-associated complex further leads to inhibition of the catalytic activity of topoisomerase II [3]. At higher concentrations, however, this inhibition may be reversed [4]. Third, doxorubicin can form free radicals, capable of damaging cancer cells. However, the importance of such free radical damage in killing tumor cells by doxorubicin is still unclear [5].

Apart from side-effects that are common to many cancer chemotherapeutics, the clinical usefulness of doxorubicin is limited largely by a cumulative dose-related cardiomyopathy that manifests itself as congestive heart failure [2]. Doxil is a doxorubicin formulation in which the drug is encapsulated in stealth liposomes that can avoid clearance by the reticuloendothelial system [6]. As a result, Doxil has a long circulation time, and it selectively extravasates through the abnormally permeable vessels characteristic of many tumors [7]. Human and animal studies demonstrate that Doxil achieves higher concentrations than doxorubicin in tumor tissue, relative to serum concentrations [8].

In this study, we genetically engineered UM-SCC-22B human head and neck squamous cancer cells with cyclic luciferase, an endogenous apoptosis marker. We applied multiple imaging strategies to monitor tumor responses to Doxil in 22B xenograft mice, including bioluminescence imaging (BLI) and diffusion-weighted magnetic resonance imaging (DW-MRI). In addition, we performed PET imaging with ${ }^{18} \mathrm{~F}-\mathrm{FDG}$ to monitor tumor glucose metabolism and ${ }^{18} \mathrm{~F}-\mathrm{FLT}$ to monitor tumor proliferation.

\section{Materials and Methods}

\section{Cell line and animal model}

pcFluc-DEVD plasmid was generously provided by Dr. T. Ozawa (Department of Chemistry, School of Science, The University of Tokyo; Japan Science and Technology Agency, Tokyo, Japan). UM-SCC-22B human head and neck squamous cells were cultured in Dulbecco's modified medium supplemented with $10 \%(\mathrm{v} / \mathrm{v})$ heat-inactivated FBS, $100 \mathrm{unit} / \mathrm{mL}$ penicillin, and $100 \mu \mathrm{g} / \mathrm{mL}$ streptomycin at $37^{\circ} \mathrm{C}$ in an at- mosphere of $5 \%(\mathrm{v} / \mathrm{v}) \mathrm{CO}_{2}$. Cells were seeded onto 6-well culture plates, transfected with pcFluc-DEVD plasmid in the presence of Lipofectamine 2000. A single clone of stably-transfected cells, denoted 22B-cFluc-DEVD, was selected by the addition of G418 (Sigma) to the culture medium for two weeks.

Six-week old female athymic mice were obtained from the NIH Division of Veterinary Resources and maintained on a standard diet at room temperature (RT). The animal experiments were approved by the Institutional Animal Care and Use Committee of the Clinical Center, NIH. Each nude mouse was injected subcutaneously with $2 \times 10^{6} 22 \mathrm{~B}-$ pcFluc-DEVD cells (in $100 \mu \mathrm{L}$ PBS) in the right shoulder. Tumor size was monitored with a digital caliper and tumor volume was calculated as $a b^{2} / 2$, where $a$ is the longest diameter and $b$ is the shortest diameter. Seven days after inoculation, 18 mice with a tumor volume around 150 $\mathrm{mm}^{3}$ were randomized into three groups $(\mathrm{n}=$ $6 /$ group). One group of mice received 1 dose of $10 \mathrm{mg} / \mathrm{kg}$ of Doxil (ALZA Corporation, USA) through intravenous administration. Another group of mice received two doses of Doxil with an interval of 2 days. The control group of mice received PBS only. Doses were selected to mimic high dose strategies used in clinical chemotherapy regimens. Animals underwent MRI, bioluminescence imaging, optical imaging and a PET scan at $48 \mathrm{hr}$ after each Doxil or PBS administration. Animal status was monitored closely throughout the experiment.

\section{Radiotracers}

${ }^{18}$ F-FDG was purchased from the Nuclear Pharmacy of Cardinal Health, and reconstituted with sterile saline. The average radiochemical purity of the product was $98.5 \%$ and specific activity was $>1,000$ $\mathrm{Ci} / \mathrm{mmol}$. Synthesis of ${ }^{18} \mathrm{~F}-\mathrm{FLT}$ was prepared manually according to methods in previous reports [9-10]. For purification of ${ }^{18} \mathrm{~F}-\mathrm{FLT}$, a semi-preparative $\mathrm{C}_{18}$ HPLC column (Phenomenex Nucleosil $\mathrm{C}_{18}$ column, $108005 ; 5 \mathrm{~mm}, 300 \times 10 \mathrm{~mm}$ ) was eluted with $8 \%$ ethanol $/ \mathrm{H}_{2} \mathrm{O}(4 \mathrm{ml} / \mathrm{min})$. Radiochemical yields (decay uncorrected) for ${ }^{18}$ F-FLT were $64.6 \pm 8.3 \%(n=5)$.

\section{MRI imaging}

Small animal MRI was conducted on a Bruker BioSpec 7.0 T system (Bruker BioSpin Corp.). The animals were placed on a holding bed equipped with circulating warm water to regulate body temperature during MRI scans. While the animals were under anesthesia, anatomic images were acquired using a $\mathrm{T}_{1}$-weighted MRI. A $\mathrm{T}_{2}$-weighted spin echo sequence (RARE) was then used with the following acquisition parameters: repetition time (TR)/echo time (TE), 
$2,000 / 48.8 \mathrm{~ms} ; 256 \times 256$ matrix; and a $30 \times 30 \mathrm{~mm}$ field of view. Continuous $1 \mathrm{~mm}$ thick slices were used to cover the entire tumor region. Diffusion weighted imaging (DWI) data were collected using a standard spin echo sequence with two b-factors (5 and 1,000 $\left.\mathrm{s} / \mathrm{mm}^{2}\right)$ in three orthogonal gradient directions $(\mathrm{x}, \mathrm{y}$, $\mathrm{z})$. The acquisition parameters were as follows: repetition time (TR)/echo time (TE), 2750/90 ms; diffusion separation time, $16 \mathrm{~ms}$; diffusion gradient duration, 6 ms; $128 \times 128$ matrix; and a $30 \times 30 \mathrm{~mm}$ field of view. A total of three to five $1 \mathrm{~mm}$-thick slices ( $1 \mathrm{~mm}$ gap) were used to cover tumor regions of interest. A magnetic resonance-compatible small animal respiratory gating device (SA Instrument, Inc.) was used during the scans.

\section{Bioluminescence Imaging}

Bioluminescence imaging was acquired with an IVIS100 imaging system (Xenogen) after MRI on each imaging day. Each mouse was injected intraperitoneally with D-luciferin at a dose of $2.5 \mathrm{mg} / \mathrm{kg}$ in $0.1 \mathrm{~mL}$. Five min later, the animals were positioned on a temperature-controlled warm bed $\left(37^{\circ} \mathrm{C}\right)$ under isoflurane anesthesia (1-2\%). Photons were collected for 180 seconds. Images were analyzed with Living Image v.3.1 software (Caliper Life Sciences).

\section{${ }^{18}$ F-FLT PET and ${ }^{18}$ F-FDG PET imaging}

For ${ }^{18} \mathrm{~F}-\mathrm{FLT}$ PET imaging the animals were administered $1.85 \mathrm{MBq}(50 \mu \mathrm{Ci}){ }^{18} \mathrm{~F}-\mathrm{FLT}$ via tail vein injection. Animals remained conscious and were allowed free access to food and water during a $1 \mathrm{~h}$ uptake period. The mice were anesthetized ( $2 \%$ isoflurane in $\mathrm{O}_{2}$ at $2 \mathrm{~L} / \mathrm{min}$ ) immediately before scanning and were imaged in the prone position for $10 \mathrm{~min}$. Body temperature was maintained before and during imaging using a thermostatically controlled circulating warm water pad.

Eight hours after ${ }^{18} \mathrm{~F}$-FLT PET imaging, the same animals received $0.85 \mathrm{MBq}(50 \mu \mathrm{Ci}){ }^{18} \mathrm{~F}-\mathrm{FDG}$ via intravenous injection. They were maintained under isoflurane anesthesia during the injection, accumulation, and scanning periods. The images were reconstructed and analyzed as reported previously [11].

\section{Histologic analysis}

Tumor samples were collected and sectioned after the animals were sacrificed. DNA fragmentation was analyzed by terminal deoxynucleotidyl transferase mediated dUTP nick end labeling (TUNEL) assay using a commercial kit (Maxin-Bio) according to the manufacturer's protocol. In random fields, the percentage of all nuclei that were TUNEL-positive nuclei was determined and used as the apoptotic index.

Frozen tumor tissue slices were fixed with cold acetone for $20 \mathrm{~min}$ and dried in the air for $30 \mathrm{~min}$ at room temperature. For staining of CD31, the slices were blocked with $1 \%$ bovine serum albumin for 30 min, incubated with rat anti-mouse CD31 antibody (1:200; BD Biosciences) and then visualized with Cy3-conjugated goat anti-rat secondary antibody (1:300; Jackson ImmunoResearch Laboratories). For Ki-67 staining, slices were incubated with rabbit anti-mouse Ki-67 antibody (1:200; BD Biosciences), and then visualized with $\mathrm{Cy} 3$-conjugated goat anti-rabbit secondary antibody (1:300; Jackson ImmunoResearch Laboratories). Both types of stained slices were washed 3 times with PBS, mounted with 4'-6-diamidino-2-phenylindole (DAPI)-containing mounting medium, and observed under an epifluorescence microscope (X81; Olympus). Each experiment was performed in pairs, and the pairs were then repeated twice. Image J software (NIH) was used to assess the total number of DAPI positive nuclei, Ki-67 positive nuclei number, and the CD31-positive area. The Ki-67 staining index (SI) was defined as the percentage of positive nuclei within the total number of nuclei. For each tumor section, ten random images were analyzed 20 times.

\section{Statistical Analysis}

Quantitative data were expressed as mean \pm SD. Statistical analysis was done using an unpaired Student's $t$ tests. $P$ values less than 0.05 were considered statistically significant.

\section{Results \\ Doxil treatment inhibits UM-SCC-22B tumor growth}

As shown in Figure 1A, a time-dependent increase in tumor volume was observed in the control group. The average tumor growth rates in the control group, expressed as $\mathrm{V} / \mathrm{V}_{0}$, were $1.35 \pm 0.27$ and $1.62 \pm$ 0.28 at day 3 and day 5 , respectively. Meanwhile, the Doxil treatment group showed $\mathrm{V} / \mathrm{V}_{0}$ ratios of $1.27 \pm$ 0.28 and $0.96 \pm 0.27$ at day 3 and day 5, respectively. No significant difference in tumor volume was observed at day 3 after 1 dose of Doxil treatment. However, a significant tumor size difference between the treatment group and the control group was observed $(p<0.01)$ on day 5 . There was no significant body weight loss observed during the treatment process.

\section{Doxil induces apoptosis in 22B tumors}

Reporter genes containing the caspase- 3 substrate peptide DEVD (aspartic acid-glutamic acid-valine-aspartic acid) have been reported to monitor caspase-3 activity when cells undergo apoptosis 
[12-15]. One example is a DEVD containing cyclic luciferase. With this method, two fragments of DnaE intein were fused to neighboring ends of firefly luciferase connected with a DEVD sequence. After translation into a single polypeptide in living cells, the amino $(\mathrm{N})$ and carboxy $(\mathrm{C})$ terminals of the luciferase were ligated by protein splicing, which resulted in a closed circular polypeptide chain. When the substrate sequence was digested by caspases, the luciferase changed into an active form and regained its activity [16]. As shown in Figure 1B and C, in the PBS-treated control tumors, only background level of BLI signal was observed. After being treated with Doxil, the BLI signal intensity increased dramatically. The results indicate increased caspase-3 activity associated with apoptosis.

\section{I8F-FDG and I8F-FLT PET imaging}

PET imaging with FDG and FLT has been well-adopted for the evaluation of tumor metabolism and proliferation. On the static FGD PET images in our study, UM-SCC-22B tumors showed clear contrast relative to surrounding tissues (other than to brain and heart). We used tumor-to-muscle ratio

(T/M) to standardize the PET quantification. At $48 \mathrm{hr}$ after 1 dose of Doxil treatment, there was no significant change of FDG uptake in treated versus control tumors (5.46 \pm 0.36 vs. $4.44 \pm 1.81, p>0.05)$. Tumors that received 2 doses of Doxil showed significantly lower FDG uptake than the baseline scan with a T/M of $2.27 \pm 0.41(p<0.01)$ (Figure 2A \& B). UM-SCC-22B tumors showed positive FLT uptake, but no significant change after treatment with 1 dose of Doxil ( $p>$ 0.05). After 2 doses of Doxil treatment, the T/M ratio dropped from $3.32 \pm 0.24$ to $2.65 \pm 0.13(\mathrm{p}<0.05)$ (Figure 3A \& B).

\section{DW-MRI}

Figure 4 shows a representative set of regular MR images, as well as the ADC map calculated from diffusion weighted images. The ADC maps revealed increases in ADC with increased Doxil dose. In the PBS control group, the average ADC value was $0.57 \pm$ $0.04 \mathrm{~mm}^{2} / \mathrm{s}$. In the Doxil treatment group, one dose of Doxil produced little change of ADC value $(0.59 \pm 0.04$ $\mathrm{mm}^{2} / \mathrm{s}$ ). After 2 doses of Doxil, however, the mean $A D C$ value increased to $0.87 \pm 0.05 \mathrm{~mm}^{2} / \mathrm{s}(\mathrm{p}<0.01)$.

A

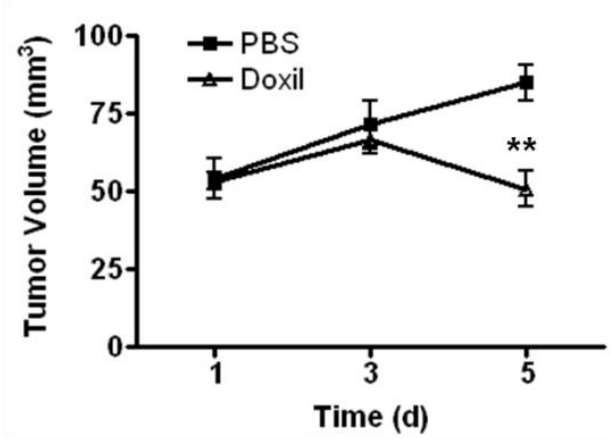

C

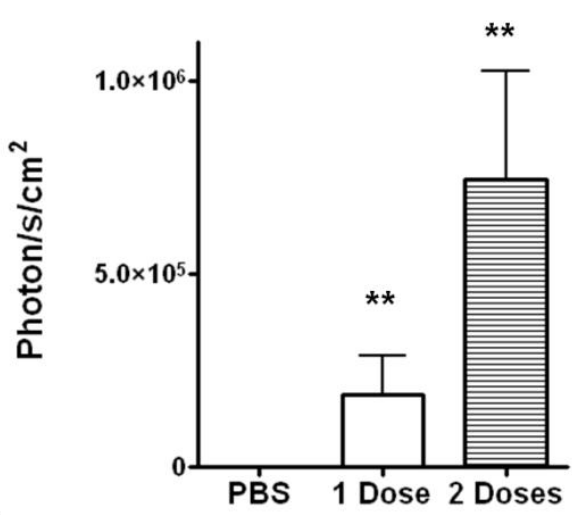

B

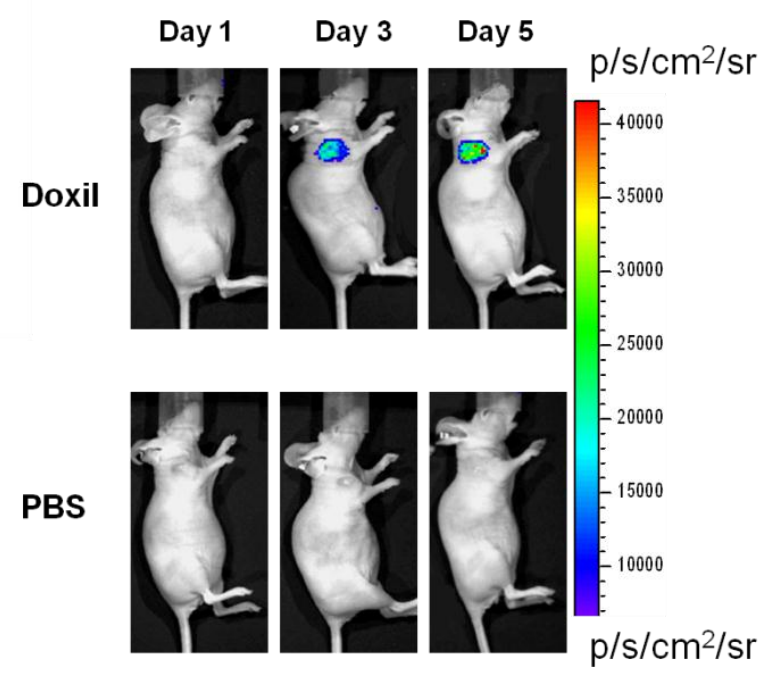

Figure I. (A) Antitumor activity of Doxil in UM-SCC-22B tumor model. The mice were implanted subcutaneously with UM-SCC-22B cells expressing apoptosis-responsive cyclic firefly luciferase (cFluc). Two doses of Doxil ( $10 \mathrm{mg} / \mathrm{kg}$ on day I, and day 3 , i.v.) inhibited tumor growth. (B) In vivo bioluminescence imaging of tumors treated with Doxil ( $10 \mathrm{mg} / \mathrm{kg})($ top) or PBS (bottom) in the UM-SCC-22B tumor bearing mice. (C) Quantification of tumor BLI intensity (**, $\mathrm{P}<0.0 \mathrm{O})$. 

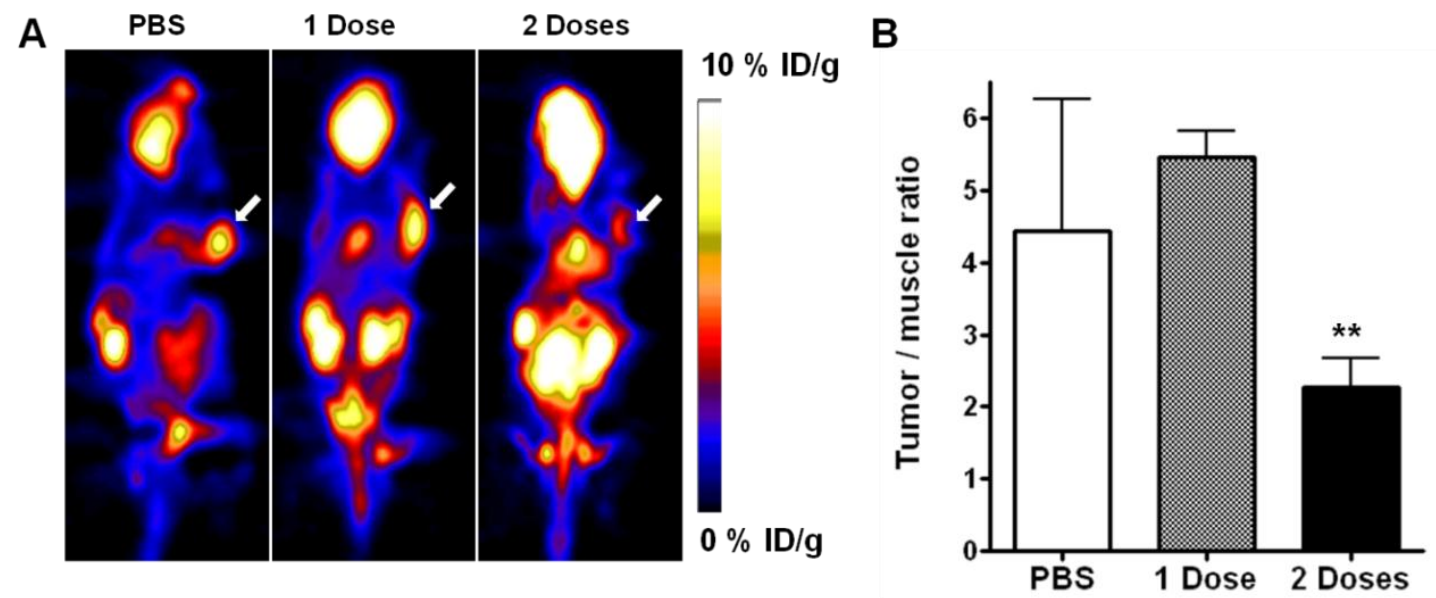

Figure 2. (A) Representative decay-corrected whole-body coronal microPET images of mice bearing UM-SCC-22B tumors at I h after intravenous injection of ${ }^{18} \mathrm{~F}$-FDG (I.85 MBq/mouse) after Doxil or PBS treatment. The tumors are indicated by arrows. (B) Tumor uptake of ${ }^{18} \mathrm{~F}-\mathrm{FDG}$ quantified by $\mathrm{ROI}$ analysis $(* *, \mathrm{p}<0.0 \mathrm{I})$.
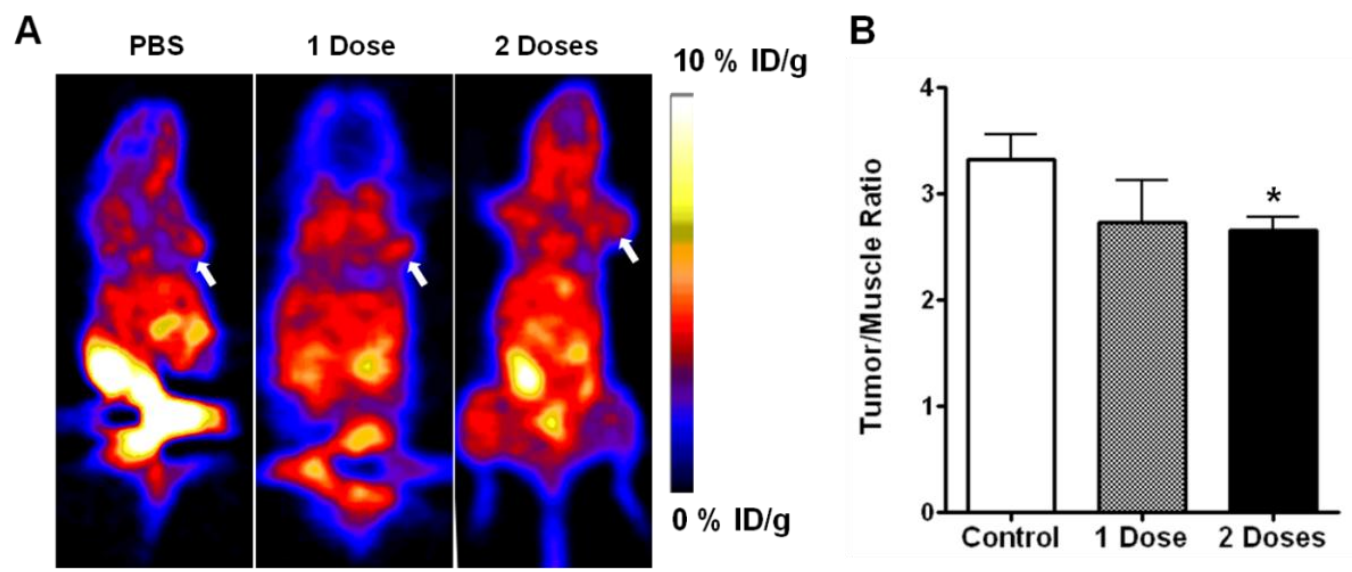

Figure 3. (A) Representative decay-corrected whole-body coronal microPET images of mice bearing UM-SCC-22B tumors at I h after intravenous injection of ${ }^{18} \mathrm{~F}-\mathrm{FLT}(\mathrm{I} .85 \mathrm{MBq} / \mathrm{mouse})$ after Doxil or PBS treatment. The tumors are indicated by arrows. (B) Tumor uptake of ${ }^{18} \mathrm{~F}-\mathrm{FLT}$ quantified by ROI analysis $(*, \mathrm{p}<0.05 ; * *, \mathrm{P}<0.0 \mathrm{I})$.

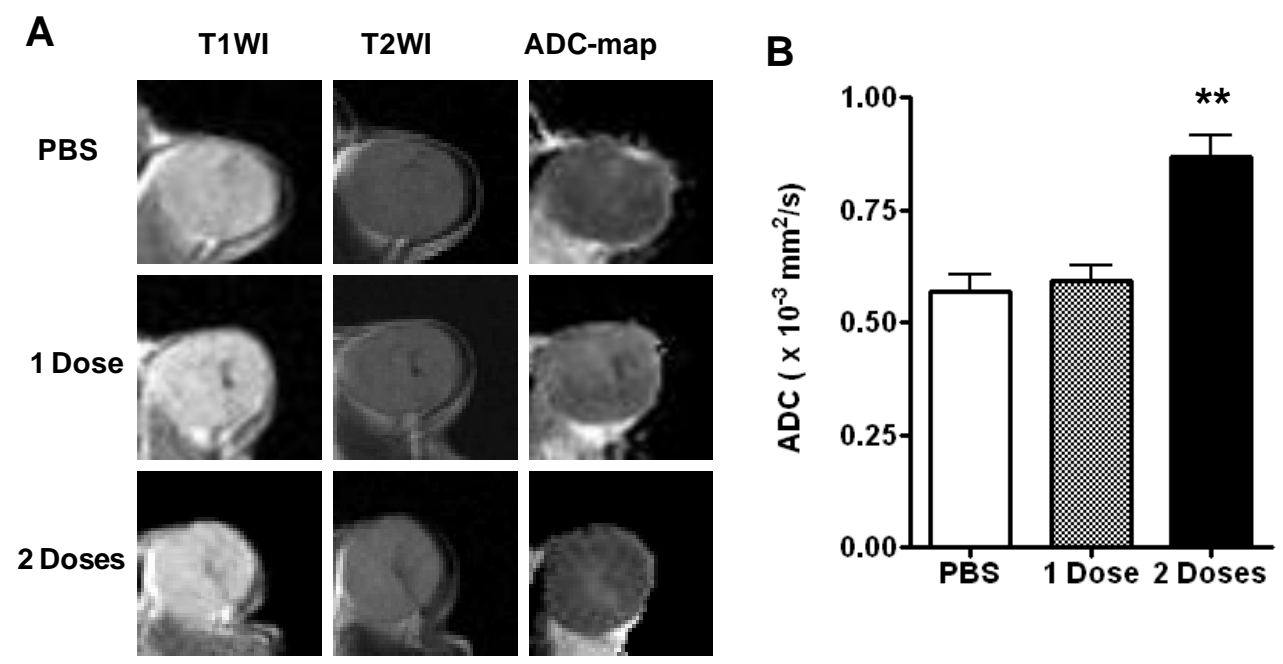

Figure 4. (A) Representative TIWI, T2WI and ADC maps of tumors treated with PBS, I dose or 2 doses of Doxil. (B) Tumor ADC quantified by region-of-interest $(\mathrm{ROI})$ analysis $(* *, \mathrm{P}<0.0 \mathrm{I})$. 


\section{Histological analysis}

The fluorescence characteristics of doxorubicin can be used to monitor the localization of the drug within lipid bilayers and liposomal delivery systems and to assess interaction of the drug with DNA and other macromolecules [17]. Even at $48 \mathrm{hr}$ after drug delivery, the presence of doxorubicin in the tumor cell nuclei could be clearly visualized by fluorescence microscopy. In addition, the drug showed homogenous distribution throughout the whole tumor (Figure 5A).

A

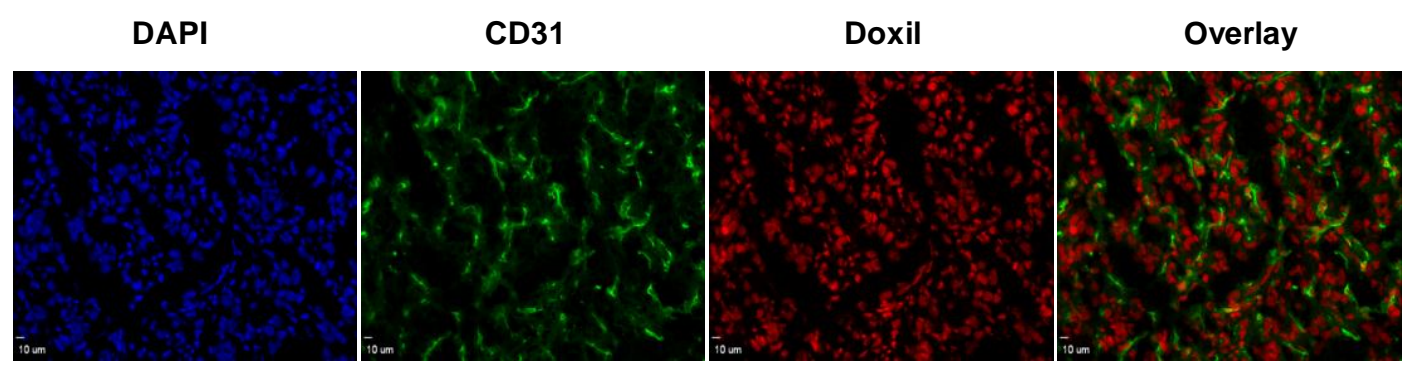

B

DAPI

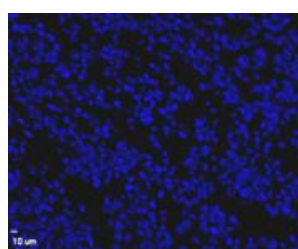

PBS
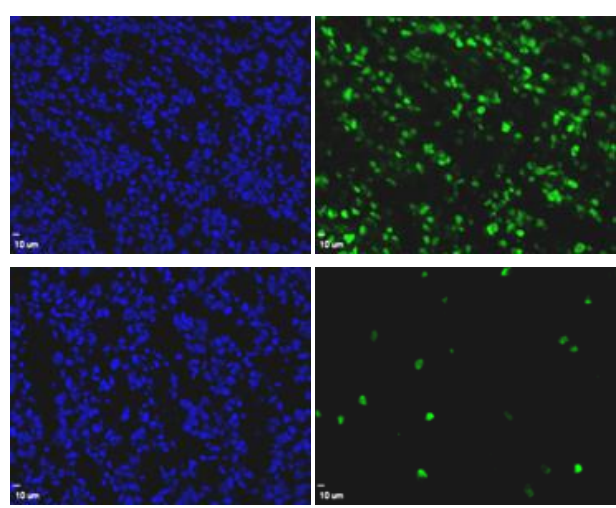

D

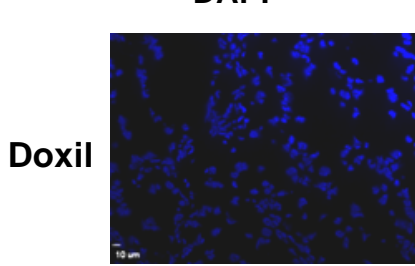

DAPI

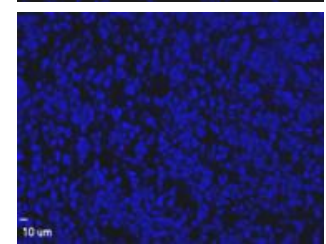

TUNEL
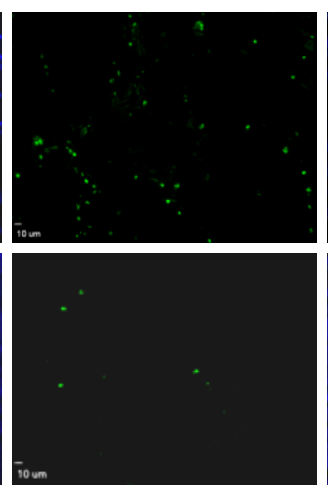

Ki67
.

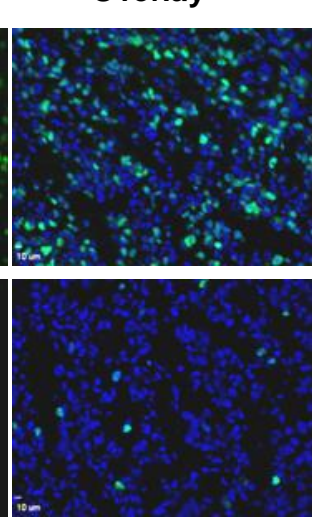

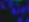

C

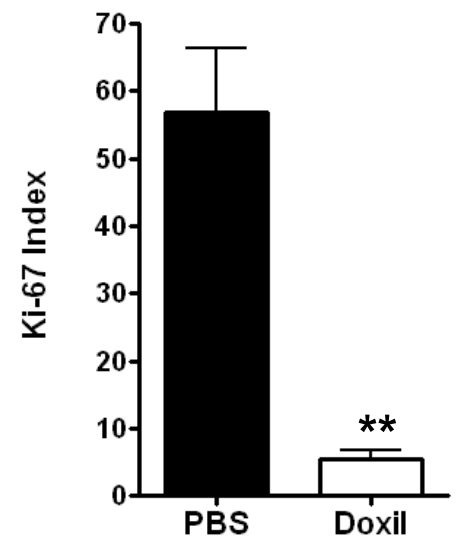

E

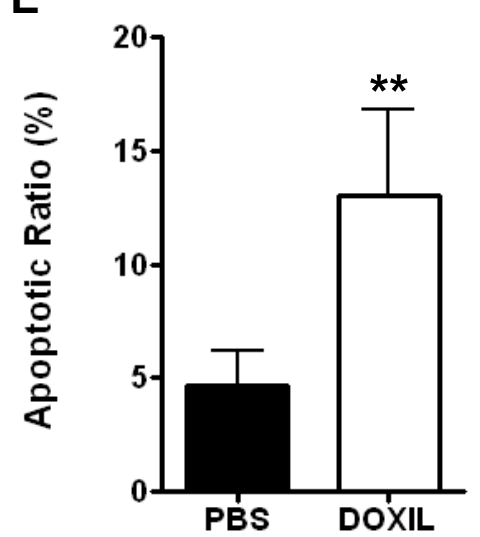

Figure 5. Histologic analyis of tumor response to Doxil. Tumor samples treated with 2 doses of Doxil (10 mg/kg) or PBS were harvested for histological analysis. (A) Immunofluorescence staining of CD3 I and fluorescence from Doxorubicin in Doxil treated tumors. (B) Proliferation of UM-SCC-22B tumor cells after Doxil treatment as assessed by Ki-67 immunofluorescence staining. (C) Ki67 positive cells were counted and the Ki-67 staining index (SI) was calculated by plotting Ki-67 positive cell number against total cell number. A significant decrease in Ki67 SI was observed after Doxil treatment, compared to the control. Three samples were used from each group, and results were confirmed with a duplicate experiment. (D) TUNEL staining of UM-SCC-22B tumor section after treatment with Doxil. Apoptotic nuclei are shown in green. Normal cell nuclei are shown in blue, stained by DAPI. (E) Apoptotic cells are counted by positive TUNEL staining, and apoptotic ratio is calculated by plotting TUNEL positive cell number against total cell number. $* *, p<0.0$ I. 
Examples of anti-Ki-67 stained sections for the controls and for the animals that received 2 doses of Doxil are shown in Figure 5B. In the control tumor sections, a relatively high percentage of cells were stained positively for Ki-67, with a Ki-67 staining in$\operatorname{dex}(\mathrm{SI})$ of $56.9 \pm 9.5 \%$. Significantly reduced cell proliferation was observed in the Doxil treated mice with a Ki-67 SI of $5.4 \pm 1.3 \%(p<0.001)$ (Figure 5 C).

A TUNEL assay is one of the traditional in vitro methods to characterize cell apoptosis, and TUNEL-positive nuclei confirm DNA fragmentation. Compared to the control group, the group that received 2 doses of Doxil treatment showed significantly more cell apoptosis, with an apoptotic index of $51 \pm 9 \%(P<0.01$, Figure 5D \& E $)$.

\section{Discussion}

In this study, we applied multiple imaging techniques to monitor tumor responses to Doxil treatment--emphasizing apoptosis-related imaging, because doxorubicin exerts its effects by inducing apoptosis [18-19]. Traditionally, apoptosis can be characterized in isolated cells by in vitro or ex vivo methods, and these histological methods play an important role if tissue can be removed from the living system by biopsy. However, potential selection bias and lack of an ability to monitor the dynamic process of apoptosis can limit the usefulness of such studies [20]. Therefore, there has been growing interest in non-invasive, functional and molecular imaging techniques to observe apoptosis longitudinally [21].

Although clinical use of optical imaging is limited by the poor penetration of light through tissues, it is useful for assessing superficial tumors, and a highly sensitive activatable probe or reporter gene approach could provide a robust platform for preclinical drug screening and therapy monitoring in small animal models [22]. To develop such a probe we began by genetically engineered UM-SCC-22B cells with an endogenous apoptosis marker, cyclic firefly luciferase. The signal from this reporter gene is triggered after caspase-3 activation. Living cells that express cyclic luciferase can be used for quantitative detection of chemicals that induce or inhibit apoptosis [16]. After Doxil treatment, the BLI signal from the cyclic luciferase increased significantly in a dose dependent manner. The results confirmed the previous finding that doxorubicin induces apoptosis via caspase- 3 activation [19]. More importantly, BLI imaging with the cyclic reporter gene is sensitive to apoptosis induced by Doxil as early as $24 \mathrm{hr}$ after a single dose of Doxil, at which time no anatomical changes are yet apparent.
Diffusion-weighted MRI (DW MRI) is a noninvasive technique that probes the random microscopic motion of water molecules in vivo [23]. If tissue water can diffuse rapidly, the ${ }^{1} \mathrm{H}$ magnetization and observed MRI signal will not refocus as much in the presence of a bipolar pulsed field gradient [24]. By systematically increasing the strength of the gradient and measuring the decrease in observed ${ }^{1} \mathrm{H}$ signal intensity, the apparent diffusion coefficient (ADC) can be determined. When $b$ values are between 0 and 1000 $\mathrm{s} / \mathrm{mm}^{2}$, DWI detects mainly extracellular space water diffusion [25]. After one dose of Doxil treatment, BLI imaging confirmed tumor cell apoptosis due to the induction of caspase protease. DWI MR detection of tumoral apoptosis relies on the observable shrinkage of the cell cytoplasm during tumor cell death, leading to decreased cell density and increased extracellular water space in the tumor, which occurs at a relatively late stage of the apoptosis process [26]. This is probably why no ADC value change was observed after only one dose of Doxil treatment.

${ }^{18} \mathrm{~F}-\mathrm{FDG}$ has been widely used both pre-clinically and clinically to monitor tumor glucose metabolism. Although treatment monitoring with ${ }^{18} \mathrm{~F}-\mathrm{FDG}$ PET may have a significant impact on patient management in multiple clinical situations, published data on the accuracy of $18 \mathrm{~F}-\mathrm{FDG}$ PET appear controversial [27]. We observed FDG signal changes after two doses of Doxil treatment, indicating the treatment reduced the tumor cell metabolism. ${ }^{18} \mathrm{~F}-\mathrm{FLT}$ is the most extensively studied probe for imaging cellular proliferation in vivo. ${ }^{18} \mathrm{~F}$-FLT PET is more specific than ${ }^{18} \mathrm{~F}-\mathrm{FDG}$ PET in evaluating tumor proliferation and tumor response, with fewer false positive findings in inflammatory lesions [28]. In our study, we did observe significantly decreased tumor uptake after 2 doses of Doxil treatment, corroborating the change of Ki-67 index.

\section{Conclusions}

Apoptosis is a very complicated and dynamic process which is still not fully understood. Multiple imaging techniques may provide a comprehensive description of apoptosis dominant tumor response to therapeutics such as Doxil. It is also essential to combine apoptosis imaging strategies with imaging of other biological or pathological pathways, such as metabolism and proliferation, to improve pre-clinical decision making in apoptosis-related diseases and interventions.

\section{Acknowledgment}

This project was supported in part by the Intramural Research Program of the National Institute 
of Biomedical Imaging and Bioengineering (NIBIB), National Institutes of Health (NIH), and the International Cooperative Program of the National Science Foundation of China (NSFC) (81028009). F. Z. and G. L. are partially supported by the NSFC grant No. 30930028. G. N. currently is an Imaging Sciences Training Program (ISTP) Fellow jointly supported by the Radiology and Imaging Sciences Department, $\mathrm{NIH}$ Clinical Center and the Intramural Research Program, NIBIB, NIH.

\section{Conflict of Interest}

The authors have declared that no conflict of interest exists.

\section{References}

1. Evan GI, Vousden KH. Proliferation, cell cycle and apoptosis in cancer. Nature. 2001; 411: 342-8.

2. Young RC, Ozols RF, Myers CE. The anthracycline antineoplastic drugs. N Engl J Med. 1981; 305: 139-53.

3. Tewey KM, Rowe TC, Yang L, Halligan BD, Liu LF. Adriamycin-induced DNA damage mediated by mammalian DNA topoisomerase II. Science. 1984; 226: 466-8.

4. Booser DJ, Hortobagyi GN. Anthracycline antibiotics in cancer therapy. Focus on drug resistance. Drugs. 1994; 47: 223-58.

5. Keizer HG, Pinedo HM, Schuurhuis GJ, Joenje H. Doxorubicin (adriamycin): a critical review of free radical-dependent mechanisms of cytotoxicity. Pharmacol Ther. 1990; 47: 219-31.

6. Smith PJ, Soues S. Multilevel therapeutic targeting by topoisomerase inhibitors. Br J Cancer Suppl. 1994; 23: S47-51.

7. Gabizon A, Catane R, Uziely B, Kaufman B, Safra T, Cohen R, et al. Prolonged circulation time and enhanced accumulation in malignant exudates of doxorubicin encapsulated in polyethylene-glycol coated liposomes. Cancer Res. 1994; 54: 987-92.

8. Northfelt DW, Martin FJ, Working P, Volberding PA, Russell J, Newman $\mathrm{M}$, et al. Doxorubicin encapsulated in liposomes containing surface-bound polyethylene glycol: pharmacokinetics, tumor localization, and safety in patients with AIDS-related Kaposi's sarcoma. J Clin Pharmacol. 1996; 36: 55-63.

9. Lee SJ, Oh SJ, Chi DY, Kil HS, Kim EN, Ryu JS, et al. Simple and highly efficient synthesis of $3^{\prime}$-deoxy-3'-[18F]fluorothymidine using nucleophilic fluorination catalyzed by protic solvent. Eur J Nucl Med Mol Imaging. 2007; 34: 1406-9.

10. Liu S, Liu Z, Chen K, Yan Y, Watzlowik P, Wester HJ, et al. ${ }^{18}$ F-labeled galacto and PEGylated RGD dimers for PET imaging of av $\beta 3$ integrin expression. Mol Imaging Biol. 2011; 12: 530-8.

11. Yang M, Gao H, Yan $Y$, Sun X, Chen $K$, Quan Q, et al. PET imaging of early response to the tyrosine kinase inhibitor ZD4190. Eur J Nucl Med Mol Imaging. 2011;[Epub ahead of print]

12. Laxman B, Hall DE, Bhojani MS, Hamstra DA, Chenevert TL, Ross BD, et al. Noninvasive real-time imaging of apoptosis. Proc Natl Acad Sci U S A. 2002; 99: 16551-5.

13. Coppola JM, Ross BD, Rehemtulla A. Noninvasive imaging of apoptosis and its application in cancer therapeutics. Clin Cancer Res. 2008; 14: 2492-501.

14. Ray P, De A, Patel M, Gambhir SS. Monitoring caspase-3 activation with a multimodality imaging sensor in living subjects. Clin Cancer Res. 2008; 14: 5801-9.
15. Kanno A, Umezawa Y, Ozawa T. Detection of apoptosis using cyclic luciferase in living mammals. Methods Mol Biol. 2009; 574: 105-14

16. Kanno A, Yamanaka Y, Hirano H, Umezawa Y, Ozawa T. Cyclic luciferase for real-time sensing of caspase-3 activities in living mammals. Angew Chem Int Ed Engl. 2007; 46: 7595-9.

17. Karukstis KK, Thompson EH, Whiles JA, Rosenfeld RJ. Deciphering the fluorescence signature of daunomycin and doxorubicin. Biophys Chem. 1998; 73: 249-63.

18. Muller I, Jenner A, Bruchelt G, Niethammer D, Halliwell B. Effect of concentration on the cytotoxic mechanism of doxorubicin--apoptosis and oxidative DNA damage. Biochem Biophys Res Commun. 1997; 230: 254-7.

19. Gamen S, Anel A, Lasierra P, Alava MA, Martinez-Lorenzo MJ, Pineiro A, et al. Doxorubicin-induced apoptosis in human T-cell leukemia is mediated by caspase- 3 activation in a Fas-independent way. FEBS Lett. 1997; 417: 360-4.

20. Massoud TF, Gambhir SS. Integrating noninvasive molecular imaging into molecular medicine: an evolving paradigm. Trends Mol Med. 2007; 13: 183-91.

21. Seddon BM, Workman P. The role of functional and molecular imaging in cancer drug discovery and development. Br J Radiol. 2003; 76: S128-38.

22. Niu G, Chen X. Has molecular and cellular imaging enhanced drug discovery and drug development? Drugs R D. 2008; 9: 351-68.

23. Le Bihan D. Molecular diffusion, tissue microdynamics and microstructure. NMR Biomed. 1995; 8: 375-86.

24. Brauer M. In vivo monitoring of apoptosis. Prog Neuropsychopharmacol Biol Psychiatry. 2003; 27: 323-31.

25. Patterson DM, Padhani AR, Collins DJ. Technology insight: water diffusion MRI--a potential new biomarker of response to cancer therapy. Nat Clin Pract Oncol. 2008; 5: 220-33.

26. Blankenberg FG, Tait JF, Strauss HW. Apoptotic cell death: its implications for imaging in the next millennium. Eur J Nucl Med. 2000; 27: 359-67.

27. Weber WA. Assessing tumor response to therapy. J Nucl Med. 2009; 50 (Suppl 1): 1S-10S.

28. Weber WA. Monitoring tumor response to therapy with ${ }^{18}$ F-FLT PET. J Nucl Med. 2010; 51: 841-4. 